\title{
The capacity and cognitive processing of vibrotactile working memory for frequency
}

\author{
Chundi Wang ${ }^{1,2,3} \cdot$ Hu Deng $^{2} \cdot$ Yu Dong ${ }^{3} \cdot$ Xuemin Zhang $^{4} \cdot$ Da-Hui Wang ${ }^{3,5}$ (D)
}

Accepted: 6 August 2021

(C) The Author(s) 2021

\begin{abstract}
A hallmark of working memory (WM) is its limited capacity. While visual and verbal domains of WM are able to store multiple items, the capacity of parametric vibrotactile WM (vtWM) has not yet been established for supra-threshold, one-dimensional sensory vibrotactile frequencies. The present study extends the standard delayed match-to-sample vibrotactile discrimination task to determine the capacity of the vtWM and its cognitive mechanism. Here, by presenting subjects with 2 to 6 vibratory frequencies sequentially in each trial, the present study demonstrates that it is possible to retain about only two vibrotactile frequencies information in vtWM. The results also showed that the capacity of vtWM does not depend on whether sequentially presented vibrotactile frequencies are delivered to the same or to different fingers. At the same time, the rate of correct report depends on sequence length and when in the sequence the stimuli are presented, suggesting the dynamic updating of vtWM similar to that of visual WM.
\end{abstract}

Keywords Vibrotactile working memory $\cdot$ Parametric working memory $\cdot$ Capacity $\cdot$ Vibrotactile frequency

\section{Introduction}

The vibrotactile working memory (vtWM) task requires the memorization of frequency of tactile vibrations (a continuous parameter), and it is considered to be a parametric WM task (Spitzer and Blankenburg 2011). Extensive electrophysiological recordings have shown that the firing rate of neurons in

Hu Deng is the Co-first author

Da-Hui Wang

wangdh@bnu.edu.cn

1 Department of Psychology and Research Centre of Aeronautic Psychology and Behavior, Beihang University, Beijing 100191, China

2 Peking University Huilongguan Clinical Medical School, Beijing Huilongguan Hospital, Beijing 100096, China

3 School of Systems Science and State Key Laboratory of Cognitive Neuroscience Science and Learning of China, Beijing Normal University, Beijing 100875, China

4 Beijing Key Laboratory of Applied Experimental Psychology, National Demonstration Center for Experimental Psychology Education (Beijing Normal University), Faculty of Psychology, Beijing Normal University, Beijing, China

5 Beijing Key Laboratory of Brain Imaging and Connectomics, Beijing Normal University, Beijing 100875, China monkey prefrontal cortex (PFC) monotonically depends on vibrotactile frequency in the vtWM task (Romo et al. 1999; Romo and Salinas 2003; Romo et al. 2012). The unique neural processing of vibrotactile frequency makes vtWM an important model system to test theories of WM and draw comparisons to other forms of WM (Wang and Wang 2021). However, although vtWM is well studied on the neural level, the cognitive and behavioral research of vtWM is still lacking. While sub-systems of WM for verbal and visual information are able to store multiple items, it is necessary to measure the capacity of vtWM. Whether vtWM can store more than one stimulus is an important question for the ability to compare vtWM to other working memory systems. This study intends to explore the capacity of vtWM and how parametric vibrotactile frequency is stored and processed in vtWM.

Modalities of the vibrotactile frequencies are different from the visual and auditory modalities. Vibrotactile frequencies are abstract (analog) features induced through a somatic sensation. Unlike visual and auditory information, which is characterized by a conjunction of features, frequency of tactile vibrations is a scalar analogue value and one-dimensional sensory stimulus. Grouping or chunking of items may influence the capacity of visual WM and rehearsal has effects on the capacity of verbal WM (Cowan 2010). But chunking or rehearsal is unlikely to affect the capacity of vtWM, since the frequency of tactile vibrations is an analog and one- 
dimensional stimulus. Thus, exploring the capacity of vtWM will improve and enrich our understanding of WM system.

Previous studies of tactile modality have investigated tactile memory capacity for location when multiple tactile stimuli were presented simultaneously to their fingers, and showed that participants can remember an average of 3.5-4.5 positions (Auvray et al. 2011; Bliss et al. 1966; Gallace and Spence 2009). However, in these studies not only tactile information but also spatial processing of that information is involved. Previous studies have also investigated tactile memory for the microgeometric qualities of tactile stimuli (such as fine textural features), and the macrogeometric qualities of tactile stimuli (i.e., object's shape, size, etc.) (Gallace and Spence 2009).

Previous vtWM studies typically applied the delayed match-to-sample vibrotactile discrimination task: subjects are presented with a vibrotactile frequency (sample) to the fingertip, then a second frequency (probe) is delivered to the same fingertip after a delay period. Subjects are instructed to compare the probe to the memorized frequency, and to decide whether the probe is of a higher or lower frequency than the sample, or whether these two vibrotactile stimuli are of the same or different frequency (Schmidt et al. 2017; Zhao et al. 2017). This standard task can be used to examine the vtWM of single frequency, but cannot be used to examine vtWM of multiple frequencies and its capacity. Although previous studies provided evidence suggestive of a multi-item storage capacity for parametric WM processing of vibrotactile frequency (Bancroft et al. 2012; Spitzer and Blankenburg 2011), the capacity has not yet been measured. Bancroft et al. (2012) used a two-item delayed match-to-sample paradigm and found results that were inconsistent with storing only one frequency in vtWM. However, the number of frequencies that vtWM can store is still unknown.

This study aims to test the capacity of vtWM and how parametric vibrotactile frequency is stored and processed in $\mathrm{vtWM}$, by extending the standard delayed match-to-sample vibrotactile discrimination task and presenting subjects with multiple vibratory frequencies sequentially in each trial. (1) Since the frequency of tactile vibrations is an analog and one-dimensional sensory stimulus, which is less unlikely to be affected by grouping or rehearsal than verbal and visual information, we hypothesized that the capacity of vtWM would be smaller than that of the verbal, visual WM, and the tactile memory for location, i.e., less than three units. (2) Previous visuospatial WM studies of sequential display showed that WM resources can be dynamically reallocated (Gorgoraptis et al. 2011). We hypothesized that processing and storage of sequentially presented vibrotactile frequency information would show similar characteristics to that of previous visuospatial WM studies. (3) In two experiments, we sequentially presented vibrotactile frequencies to the same fingertip and to different fingertips to test the capacity and processing of vtWM. Evidence from previous studies indicated that when stimuli are retained in both the sensory cortex and the anterior regions like PFC, interference among stimuli representations may decrease and capacity may improve (Christophel et al. 2017). Thus, if the secondary somatosensory cortex contributes to vtWM capacity, frequencies delivered to different fingertips might improve vtWM capacity.

\section{Experiment 1: Sequential Presentation to the Same Fingertip}

In experiment 1 , vibrotactile frequencies were sequentially presented to the same fingertip to test the capacity and processing of vtWM for frequency.

\section{Methods}

\section{Participants}

A total of 19 (11 females) right-handed healthy undergraduate and graduate students (age range: 18 years to 26 years) participated in Experiment 1. The sample size was determined a priori from a power analysis ( $G^{*}$ Power), which indicated that this number of participants was sufficient to detect a withinsubjects effect $\left(\eta_{p}{ }^{2}=0.2\right) 90 \%$ of the time using an alpha $(\alpha)$ of 0.05 . The effect size is based on pervious visual and vtWM study, and also a pilot experiment (Bancroft et al. 2012; Spitzer and Blankenburg 2011; Gorgoraptis et al. 2011). All of the participants self-reported normal tactile perception. All participants gave written consent prior to the experiment and received payment after the experiment. This study was approved by the Institutional Review Board and Ethics Committee of Human Participant Protection.

\section{Stimuli}

Vibrotactile stimuli are generated by a Braille-like display of 8 pins ( $2 \times 4$ matrix with $2.5 \mathrm{~mm}$ spacing). The vibrotactile frequency generators were controlled by a programmable piezoelectric mechanical stimulator (QuaeroSys, St. Johann, Germany). All 8 pins of the Braille-like display vibrated with equal amplitude and intensity, with smoothed onsets and offsets. Five vibrotactile frequency generators were installed in a hand brace, and each of them provided frequencies to each of the five fingertips (Fig. 1).

To test the capacity of vtWM and avoid the effect of individual differences, this study recruited participants to assess the frequencies to make sure that the frequencies used in the present study are highly discriminable and supra-threshold for all participants. The frequencies in the present study were selected from the range of $10-70 \mathrm{~Hz}$ based on previous behavioral and imaging studies of vtWM (Bancroft et al. 2012; 

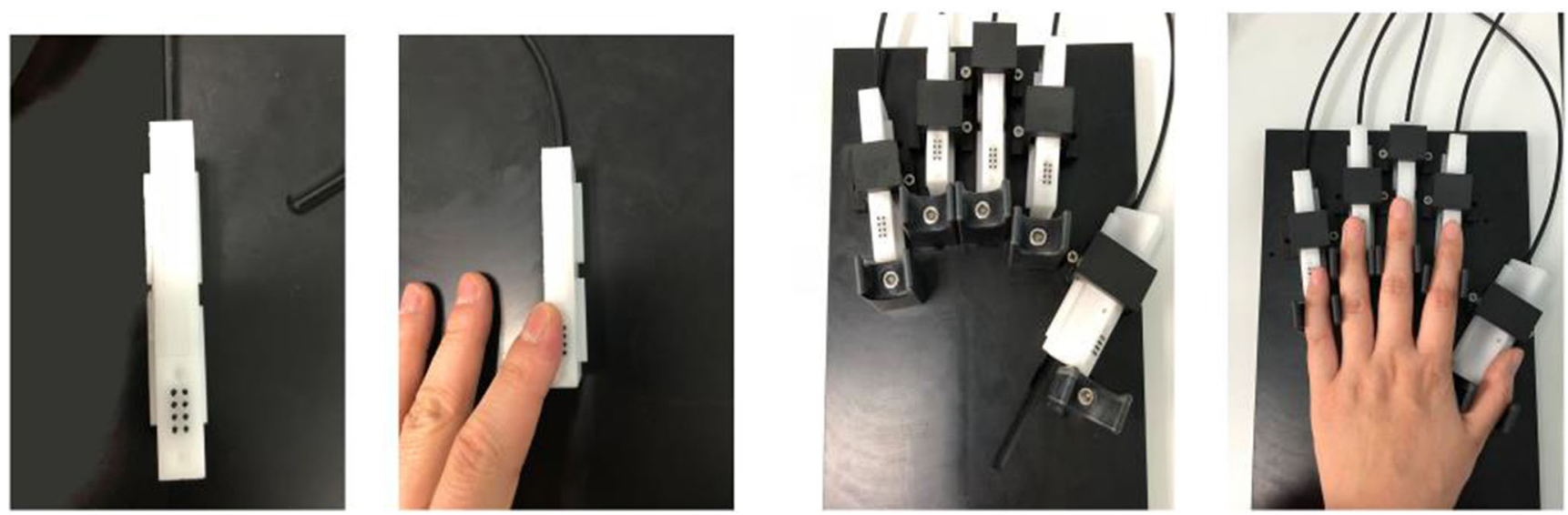

Fig. 1 Vibrotactile stimulator (QuaeroSys, St. Johann, Germany) and custom hand brace

Spitzer and Blankenburg 2011; Schmidt et al. 2017; Zhao et al. 2017). Before the experiment, we recruited participants to assess the frequencies. Subjects were presented with two different $1000 \mathrm{~ms}$ vibratory frequencies, separated by an unfilled $500 \mathrm{~ms}$ period. Subjects were instructed to indicate whether the second stimulus was higher or lower than the first stimulus. The set of frequencies of 10, 20, 30, 40, 50, 60, and $70 \mathrm{~Hz}$ were tested, and accuracies for each pair of these frequencies were above $95 \%$. Thus, the set of discriminable frequencies of $10,20,30,40,50,60$, and $70 \mathrm{~Hz}$ were used in the present study.

\section{Procedure}

The procedure for measuring vtWM capacity in this study was designed based on previous vtWM and visuospatial WM studies (Gorgoraptis et al. 2011; Hernández et al. 2010; Romo et al. 1999; Romo et al. 2012; Schmidt et al. 2017; Zhao et al. 2017). The one-item delayed match-to-sample paradigm used in previous vtWM studies cannot be used to examine vtWM of multiple frequencies and its capacity. Therefore, this study referred to the method of measuring capacity in visual tasks to improve this paradigm, presenting subjects with multiple vibratory frequencies sequentially in each trial (Gorgoraptis et al. 2011). Using this new procedure, the present study was able to test participants' performance of remembering multiple frequencies using single-probe report, which is often used in the visuospatial WM capacity. Then based on the accuracy of the report, this study estimated the number of memorized vibrotactile frequencies by following a standard formula used to assess single-probe capacity (Cowan 2001).

The vibration frequencies of tactile stimuli were selected from 10, 20, 30, 40, 50, 60, and $70 \mathrm{~Hz}$. Vibrotactile stimuli were delivered to the left index finger. Subjects were presented with two, three, four, five, or six different vibratory frequencies, separated by an unfilled $500 \mathrm{~ms}$ period. The duration of each stimulus presentation was $1000 \mathrm{~ms}$. The set size varied from 2 to 6 . The serial number (" 1 ", " 2 ", “3", "4", "5",
"6") of the sample stimuli were presented on the computer screen simultaneously with the vibrotactile stimuli. The serial number was only used as a marker, and for achieving the goal of testing the vtWM of each frequency in the sequence. Just like previous visuospatial WM capacity studies used color to identify the stimuli (Gorgoraptis et al. 2011). The serial number in this study played the same role of marker as color in previous visuospatial WM studies.

Participants were required to remember all the vibratory frequencies. After all vibratory stimuli were presented and a $500 \mathrm{~ms}$ delay period was provided, a randomly selected serial number was presented on the screen, and a probe frequency was delivered to the same fingertip. Participants were required to indicate whether the probe was identical to the frequency cued by the number presented on the screen (Fig. 2). The probe frequency was either the same frequency as the sample delivered to the probe finger or a new frequency that was not presented in the sample array. Responses were given via right middle and index finger button presses. And identical and different probe frequencies occurred randomly at a probability of 0.5 , respectively. All frequencies in the sequence were equally likely to be tested. Each subject completed a total of 400 interleaved trials. There were 20 trials for each of the 20 combinations of sequence length (2-6) and serial position of the probe frequency within the sequence.

The experimental procedures were programmed in MATLAB R2012b (The Math Works) using the Psychophysics Toolbox. Participants were tested individually in a room. The sound of the stimulator was masked by white noise $(90 \mathrm{~dB})$ presented through headphones. The volume was adjusted, and none of the participants reported hearing any residual sound of the vibrotactile stimulation. During the practice session, participants were provided with feedback on their accuracy. All participants answered one question ("What strategy did you use to remember the frequencies?") after participating in Experiment 1. 


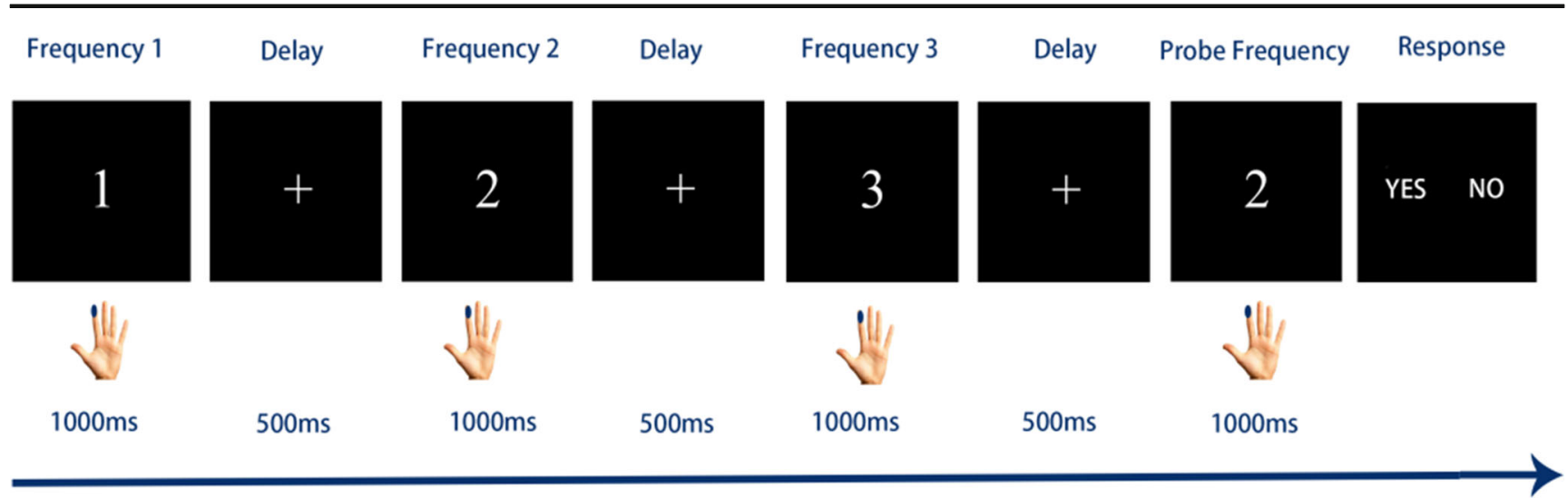

Fig. 2 Sample trial sequence of Experiment 1 (sequentially presented frequencies delivered to the same fingertip)

\section{Results and Discussion}

The accuracies of different set size conditions are shown in Fig. 3A. The main effect of set size was significant, $F_{(4,72)}=$ 17.491, $p<0.001, \eta_{\mathrm{p}}{ }^{2}=0.493$. The pairwise comparison (Bonferroni corrected) between the five conditions further indicated that two vs. four frequencies conditions, $\mathrm{MD}=0.090$, $p=0.002$; two vs. five frequencies conditions, $\mathrm{MD}=0.089$, $p=0.002$; two vs. six frequencies conditions, $\mathrm{MD}=0.167$, $p<0.001$; and three vs. six frequencies conditions, $\mathrm{MD}=$
$0.121, p<0.001$, were significantly different from each other. The d' (d-prime) of different set size conditions are shown in Fig. 3B. The main effect of set size was significant, $F_{(4,72)}=$ 25.73, $p<0.001, \eta_{\mathrm{p}}{ }^{2}=0.59$. The pairwise comparison (Bonferroni corrected) between the five conditions further indicated that two vs. four frequencies conditions, $\mathrm{t}_{(18)}=6.03$, $p<0.001$, Cohen's $\mathrm{d}=1.38$; two vs. five frequencies conditions, $\mathrm{t}_{(18)}=6.06, \mathrm{p}<0.001$, Cohen's $\mathrm{d}=1.39$; two vs. six frequencies conditions, $\mathrm{t}_{(18)}=7.99, \mathrm{p}<0.001$, Cohen's $\mathrm{d}=$ 1.83; three vs. five frequencies conditions, $\mathrm{t}_{(18)}=4.11, p=$

$\mathrm{B}$

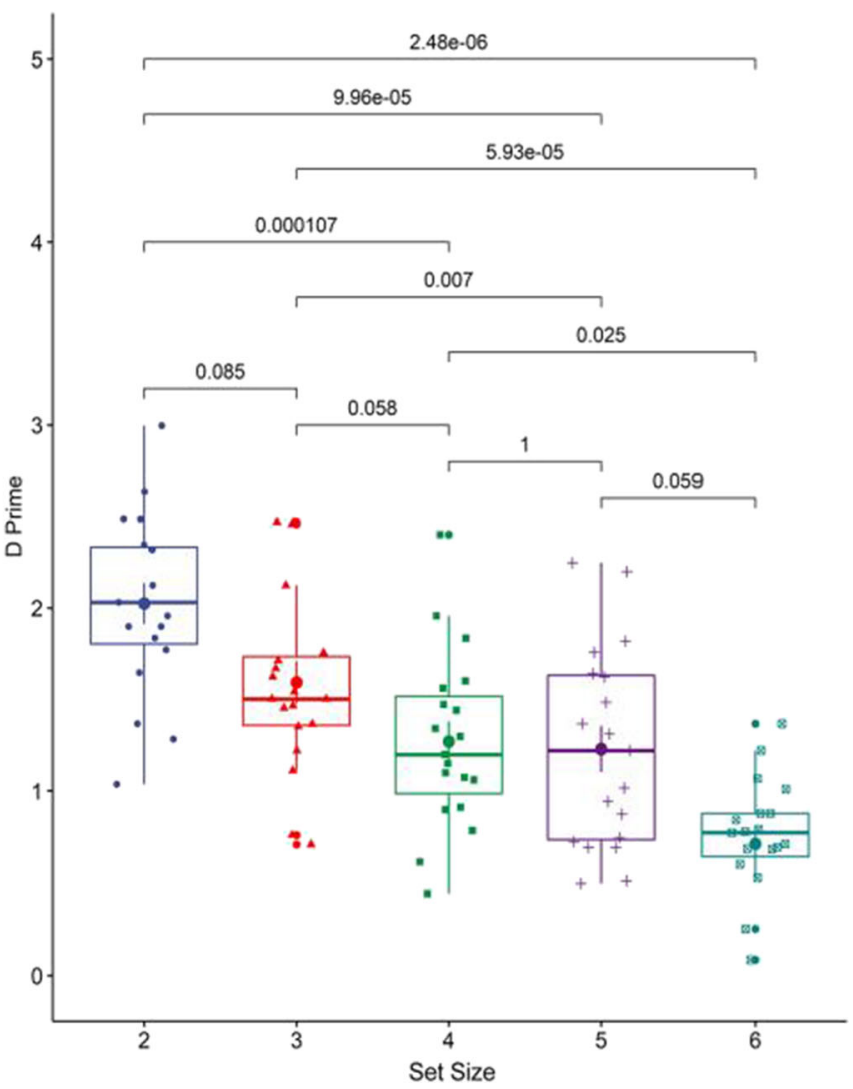

Fig. 3 (A) Accuracies of five set size conditions in Experiment 1. (B) The d' (d-prime) of different set size conditions in Experiment 1. The error bars represent standard error of the mean 
0.007, Cohen's d $=0.94$; three vs. six frequencies conditions, $\mathrm{t}_{(18)}=6.32, \mathrm{p}<0.001$, Cohen's $\mathrm{d}=1.45$, and four vs. six frequencies conditions, $\mathrm{t}_{(18)}=3.51, p=0.025$, Cohen's $\mathrm{d}=0.80$, were significantly different from each other.

Figure 4 shows how accuracy varied as a function of its serial position for different set sizes (sequence lengths). There was a recency effect, and the last item was remembered with the most accuracy. Serial order had a significant effect on accuracy (main effect of serial order $F_{(3.20,57.53)}=8.82$, $p<0.001, \eta_{\mathrm{p}}{ }^{2}=0.33$, Greenhouse-Geisser), regardless of the total number of frequencies in the sequence with the most recent frequency remembered significantly more accurately than the preceding frequencies. The main effect of set size for the last item in a sequence was not significant, $F_{(2.55,45.96)}=0.68, p=$ $0.545, \eta_{\mathrm{p}}{ }^{2}=0.04$ (Greenhouse-Geisser). The accuracy of the recall of the most recent frequency was unaffected as the total number of items held in memory increased. The main effect of the set size for the second to last item in a sequence was significant, $F_{(2.54,45.75)}=4.90, p=0.007, \eta_{\mathrm{p}}{ }^{2}=0.21$ (GreenhouseGeisser). Accuracy in the recall of the second to last item significantly decreased with the increase of the sequence length. Performance was significantly better than chance for every combination of serial order and set size $\left(t_{\mathrm{s}}>8.10, \mathrm{df}_{\mathrm{s}}=18, p_{s}<0.001\right.$, Cohen's $d_{s}>1.86$ ), indicating that some information was stored about every position in a sequence.

Based on the accuracy of report, this study estimated the number of memorized vibrotactile frequencies by following a standard formula used to assess single-probe capacity (Cowan 2001): $\mathrm{K}=\mathrm{N}^{*}(\mathrm{H}-\mathrm{FA})$, where $\mathrm{H}$ and $\mathrm{FA}$ are observed hit and false alarm rates, and $\mathrm{K}$ is the number of memorized items and $\mathrm{N}$ is the set size of the stimuli. The results in Fig. 5A show that the number of memorized frequencies increase at first and then decreases along with the set size. The maximum number of memorized frequencies is 2.1 given sequentially presented frequencies. Further, we calculated capacity with the last item excluded from each set size condition (Fig. 5B). The maximum number of memorized frequencies with the last item excluded is 1.9. We collected all participants' answers about the strategies that they had taken. None of them mentioned using any explicit grouping or rehearsal strategies.

The results suggested that the memory capacity of vtWM is 2 units when vibratory frequencies are sequentially delivered to the same fingertips. Performance was significantly better than chance for each frequency of serial order and set size, indicating that some information was stored about every frequency in a sequence. The mean accuracy of each frequency was recalled declined with increases in total vtWM load, but also depended on when in the sequence it appeared. All these results suggested that vtWM resources allocation to each new frequency to be remembered is affected by sequence length and serial order dynamically. Thus, suggesting the dynamic updating of vtWM similar to that of visual WM of sequential display, which means that resources can be dynamically reallocated in vtWM for frequency of each serial order and set size.

\section{Experiment 2: Sequential Presentation to Different Fingertips}

Previous studies found the engagement of PFC and somatosensory cortex during vibrotactile frequency maintenance and processing (Harris et al. 2001; Harris et al. 2002; Spitzer and Blankenburg 2011; Zhao et al. 2017). Evidence from previous studies suggested that when stimuli were retained in the sensory cortex and the anterior regions, interference among stimuli representations might decrease and capacity might improve (Christophel et al. 2017). In the hand representation of the secondary somatosensory cortex, most neurons have independent receptive fields (Merzenich et al. 1978; Iwamura et al. 1993). Thus, if the secondary somatosensory cortex contributes to vtWM capacity, frequencies delivered to different fingertips might improve vtWM capacity. Therefore, we further
Fig. 4 Accuracies modulated by serial order and sequence length in Experiment 1. Accuracy is plotted against order in the sequence. The error bars represent standard error of the mean

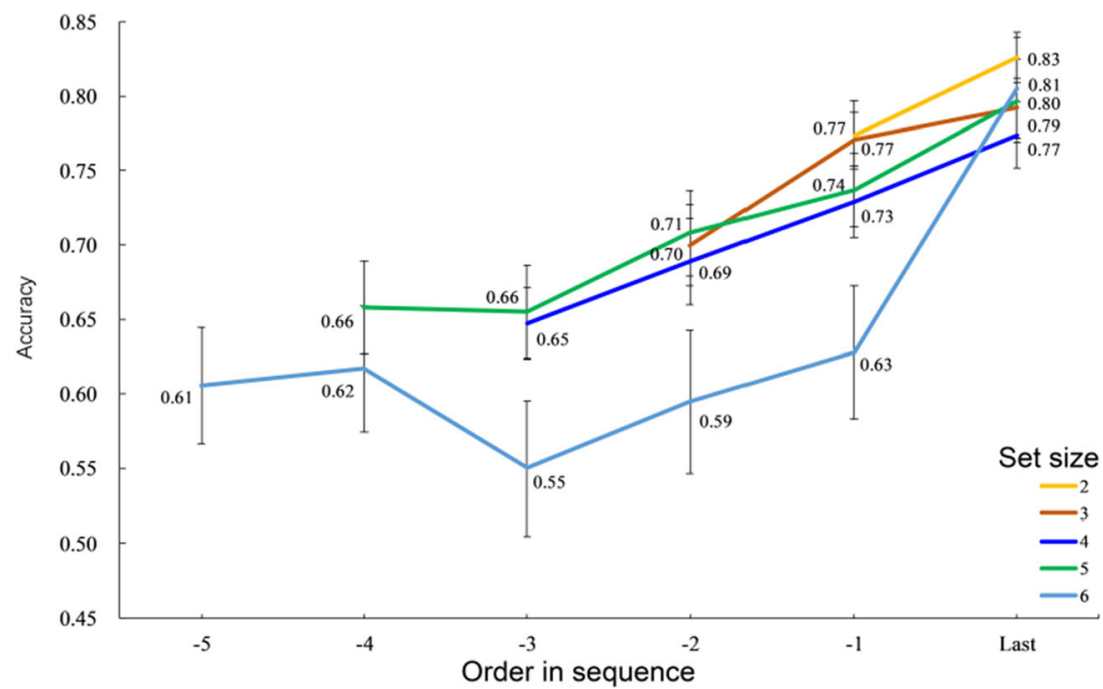


A

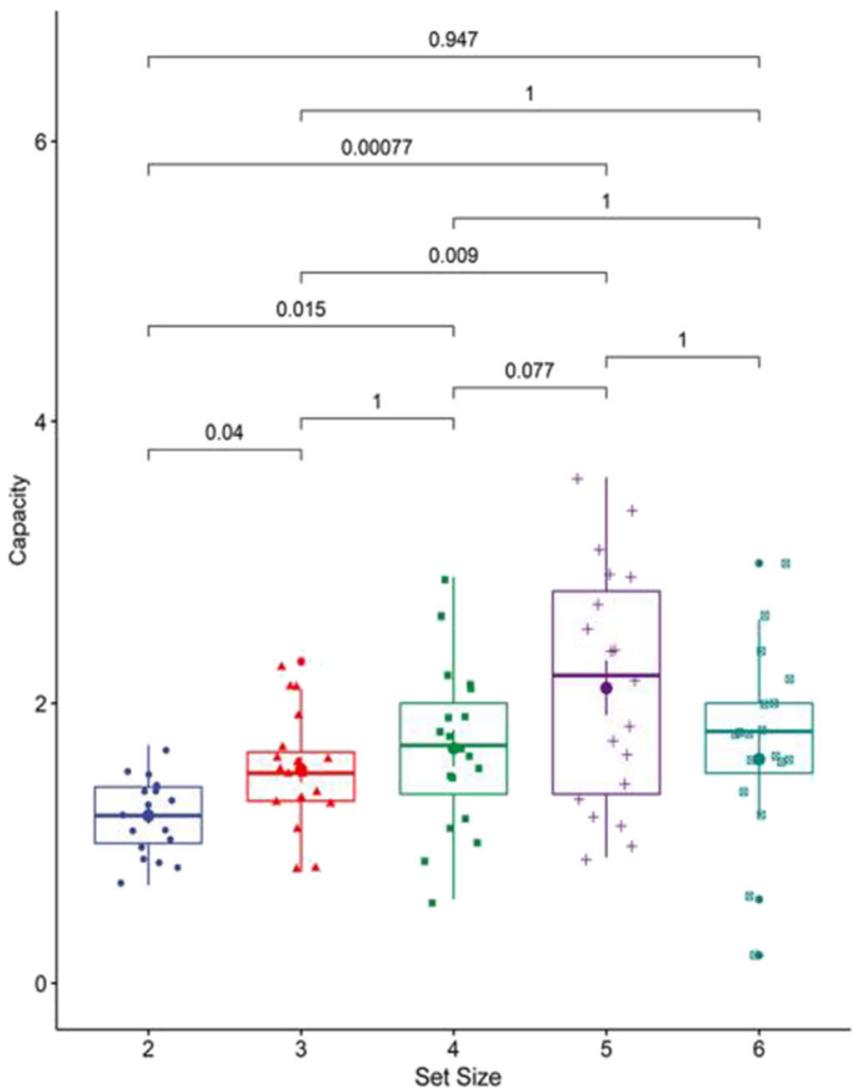

B

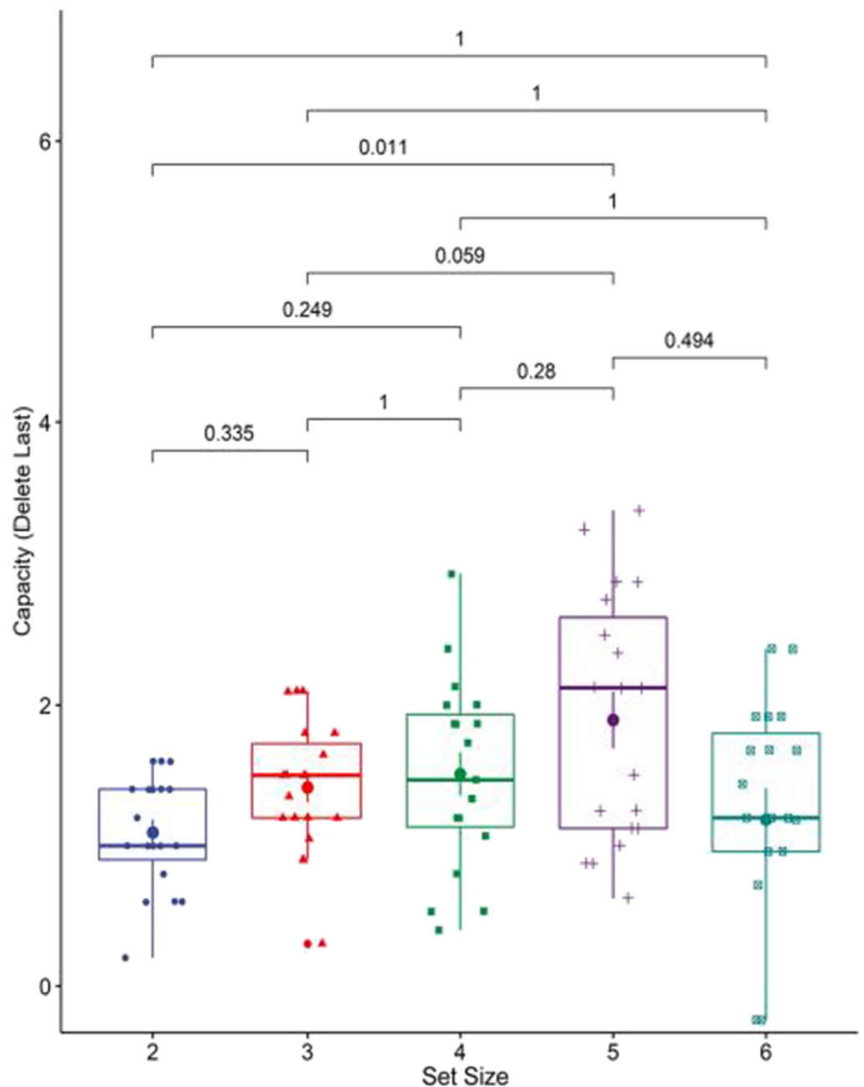

Fig. 5 (A) Capacity of five set size conditions in Experiment 1. (B) Capacity of five set size conditions with the last item excluded in Experiment 1. The error bars represent standard error of the mean

delivered samples to different fingertips in Experiment 2 to test the vtWM capacity (Fig. 6).

\section{Methods}

\section{Participants}

A total of 19 (10 females) right-handed healthy undergraduate and graduate students (age range: 18 years to 26 years) participated in Experiment 2. All of the participants selfreported normal tactile perception. All participants gave written consent prior to the experiment and received payment after the experiment. This study was approved by the Institutional Review Board and Ethics Committee of Human Participant Protection.

\section{Procedure}

Vibration frequencies of tactile stimuli were selected from 10 , 20, 30, 40, 50, 60, and $70 \mathrm{~Hz}$. Subjects were presented with

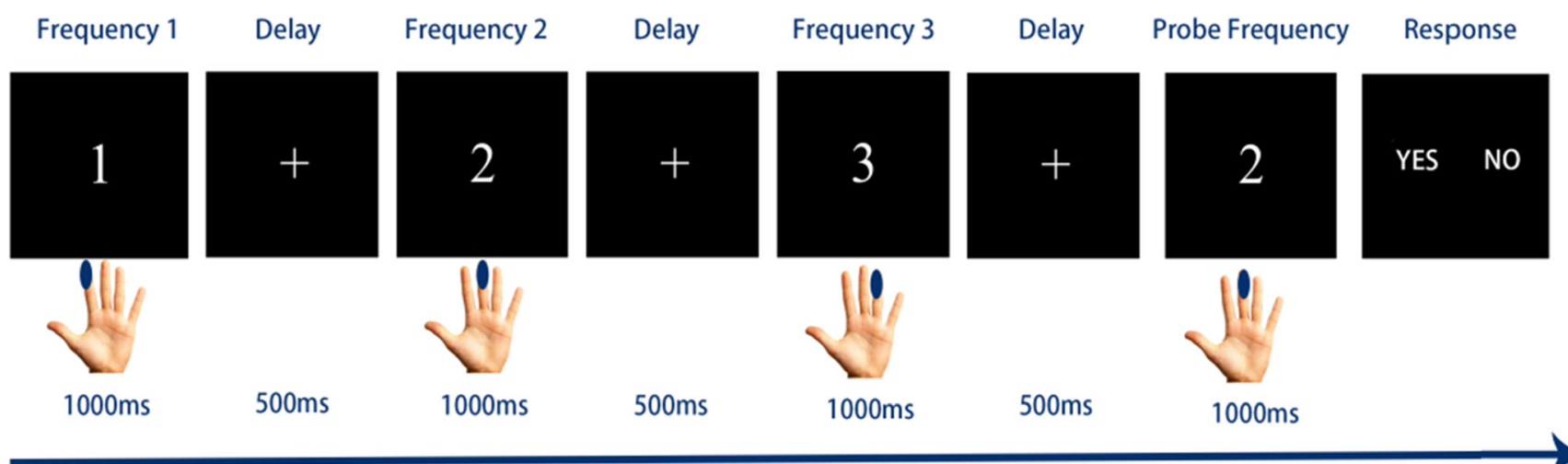

Fig. 6 Sample trial sequence of Experiment 2 (sequentially presented frequencies delivered to different fingertips) 
two, three, four, or five different vibratory frequencies sequentially, separated by an unfilled $500 \mathrm{~ms}$ period. Each frequency was sequentially delivered to each different finger of the left hand. We delivered two tactile stimuli sequentially to the index finger and middle finger; three frequencies to the index finger, middle finger, and ring finger; four frequencies to the index finger, middle finger, ring finger, and little finger; and five frequencies to all five fingers of the left hand respectively (Fig. 6).

The set size varied from 2 to 5 . When the vibratory stimulus was presented, the serial number ("1", " 2 ", " 3 ", “4”, " 5 ") of the stimulus was simultaneously presented on the screen. Participants were instructed to remember all the vibratory frequencies. After the presentation of vibratory stimuli, a randomly selected serial number was presented on the screen to indicate which one sample would be tested, and a probe frequency was delivered to the finger that the delivered frequency was selected to test. Each subject completed a total of 280 interleaved trials. There were 20 trials for each of the $14 \mathrm{com}-$ binations of sequence length (2-5) and serial position of the probe frequency within the sequence. All participants answered one question ("What strategy did you use to remember the frequencies?") after participating in Experiment 2.

\section{Results and Discussion}

The results of Experiment 2 are similar to those of Experiment 1. The accuracies of the four conditions are shown in Fig. 7A. The main effect of set size was significant, $F_{(3,54)}=11.049$, $p<0.001, \eta_{\mathrm{p}}{ }^{2}=0.380$. The pairwise comparison (Bonferroni corrected) between the four conditions further indicated that two vs. four frequencies conditions, $\mathrm{MD}=0.069, p=0.003$; two vs. five frequencies conditions, $\mathrm{MD}=0.074, p<0.001$; and three vs. five frequencies conditions, $\mathrm{MD}=0.046, p=$ 0.035 , were significantly different from each other. The d' (d-prime) of different set size conditions are shown in Fig. 7B. The main effect of set size was significant, $F_{(3,54)}=$ 15.73, $p<0.001, \eta_{\mathrm{p}}{ }^{2}=0.47$. The pairwise comparison (Bonferroni corrected) between the four conditions further indicated that two vs. four frequencies conditions, $t_{(18)}=$ 4.46, $p=0.002$, Cohen's $d=1.02$; two vs. five frequencies conditions, $\mathrm{t}_{(18)}=7.07, \mathrm{p}<0.001$, Cohen's $\mathrm{d}=1.62$; and three vs. five frequencies conditions, $\mathrm{t}_{(18)}=3.99, p=0.005$, Cohen's $d=0.91$ were significantly different from each other.

Serial order had a significant effect on accuracy (main effect of serial order $F_{(2.63,47.39)}=11.44, p<0.001, \eta_{\mathrm{p}}{ }^{2}=0.39$, Greenhouse-Geisser), regardless of the total number of fre-
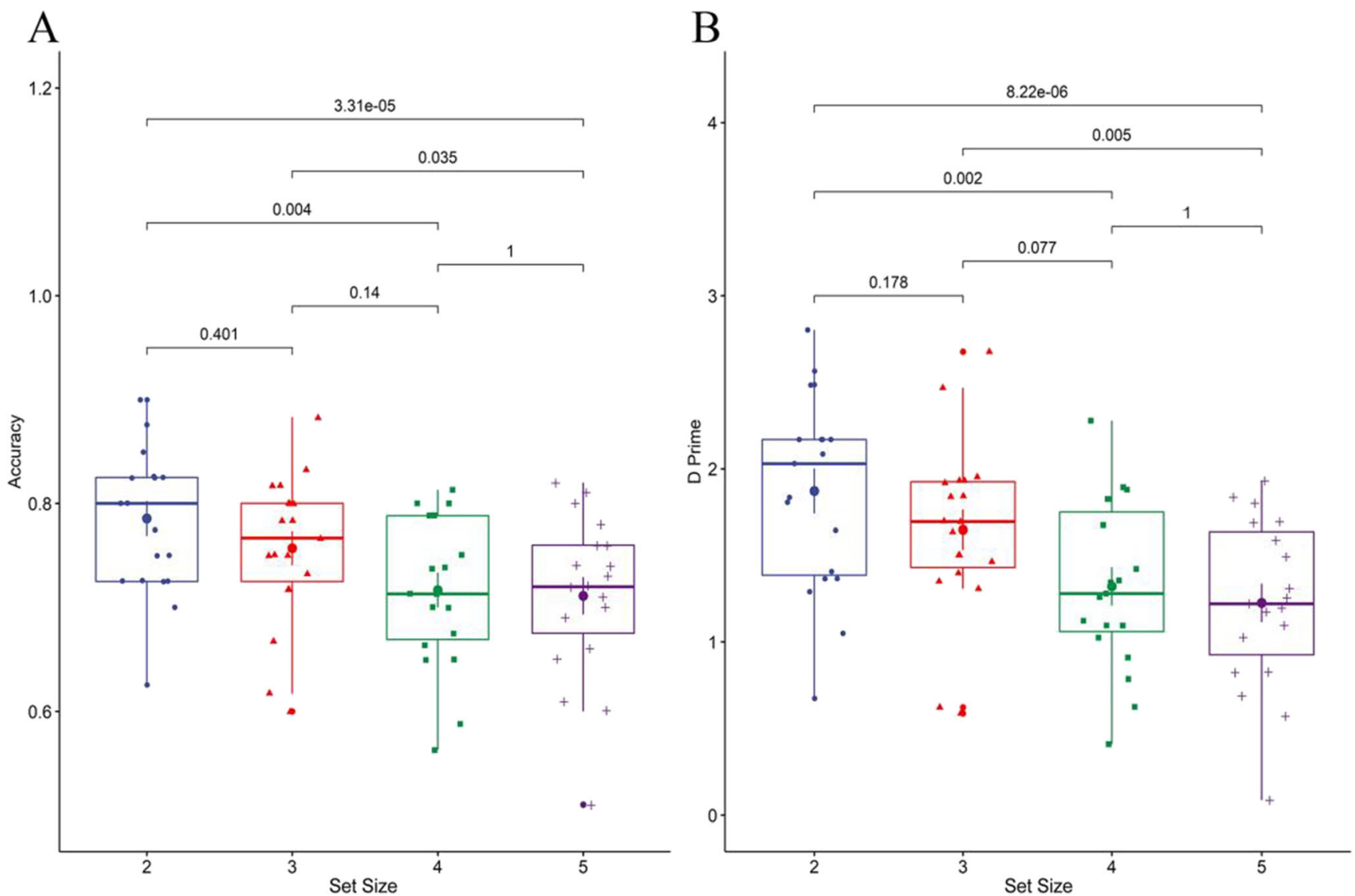

Fig. 7 (A) Accuracies of the four set size conditions in Experiment 2. (B) The d' (d-prime) of different set size conditions in Experiment 1. The error bars represent standard error of the mean 
quencies in the sequence, with the most recent frequency being remembered significantly more accurately than the preceding frequencies. Thus, there was a clear recency effect (Fig. 8). Similar to the results of Experiment 1, the main effect of set size for the last item in a sequence was not significant, $F_{(3,54)}=0.57, p=0.635, \eta_{\mathrm{p}}{ }^{2}=0.03$. Accuracy in the recall of the last item was not significantly affected by sequence length, and thus, the memorized items in vtWM had no effect on the most recent frequency. The main effect of set size for the second to last item in a sequence was significant, $F_{(3,54)}=$ $3.52, p=0.021, \eta_{\mathrm{p}}{ }^{2}=0.16$. Accuracy for the second to last item decreased significantly as the number of preceding items increased. Performance was significantly better than chance for every combination of serial order and set size $\left(t_{\mathrm{s}}>5.90\right.$, $\mathrm{df}_{\mathrm{s}}=18, p_{s}<0.001$, Cohen's $d_{s}>1.35$ ), indicating that some information was stored about every position in a sequence.

The present study also calculated the number of memorized frequencies. The results are shown in Fig. 9A. The maximum number of memorized frequencies is 2.1. Further, we calculated capacity with the last item excluded (Fig. 9B). The maximum number of memorized frequencies with the last item excluded is 1.94. In the questionnaire that we collected, none of the participants mentioned using any explicit grouping or rehearsal strategies.

Similar results were found when frequencies are sequentially delivered to the same fingertip in Experiment 1 and to different fingertips in Experiment 2. The results in Experiment 2 indicated that $\mathrm{vtWM}$ can store about two frequencies when vibratory frequencies are sequentially delivered to different fingertips. The results of Experiment 2 confirmed the conclusions of Experiment 1, that the capacity of vtWM is two units and the resources can be dynamically reallocated in vtWM. The processing and storage of vibrotactile frequency information of vtWM does not depend on whether sequentially presented frequencies are delivered to the same or to different fingers. These results suggested that the processing and storage of vtWM may be mainly contributed by the higher cortex like the PFC.

\section{Discussion}

In summary, our findings indicate the following. 1) vtWM can hold two sequentially delivered vibrotactile frequencies. 2) Information stored in vtWM is dynamically updated when vibrotactile stimuli are sequentially delivered. The length of the sequence has no significant effect on the accuracy of the last item but has a significant effect on the rest of the items. Finally, 3) The capacity of vtWM does not depend on whether sequentially presented vibrotactile frequencies are delivered to the same or to different fingers.

First, the capacity of vtWM for vibrotactile frequency is two units. The present study shows a multi-item storage capacity for vtWM, which lays the foundations for comparing the vtWM to other working memory systems. By presenting subjects with multiple vibratory frequencies sequentially in a trial, we found a multi-item storage capacity for vtWM processing of vibrotactile frequency. Previous studies of tactile memory for location showed that participants can remember about 4 positions presented simultaneously to their fingertips (Auvray et al. 2011; Bliss et al. 1966). Different from spatial information, frequency of tactile vibrations is a scalar abstract value and one-dimensional sensory stimulus. Thus, chunking or rehearsal is unlikely to affect the capacity of parametric $\mathrm{WM}$ of vibrotactile frequency. In the questionnaire that we collected after experiments, none of the participants reported using an explicit grouping or rehearsal strategy. Most of the participants reported that it was difficult to memorize the vibrotactile frequencies, and no strategy was available. The present study showed that the parametric vtWM can store multiple frequencies, and the capacity of vtWM is smaller than previous tactile memory capacity studies for location.

Second, vtWM resources for sequential frequency stimuli is dynamically updated, which is similar to observations in the visuospatial WM of sequential display (Gorgoraptis et al. 2011). The results showed that serial order had a significant effect on accuracy, regardless of the total number of frequencies in the sequence with the most recent frequency
Fig. 8 Accuracies modulated by serial order and sequence length in Experiment 2. Accuracy is plotted against order in the sequence. The error bars represent standard errors of the mean

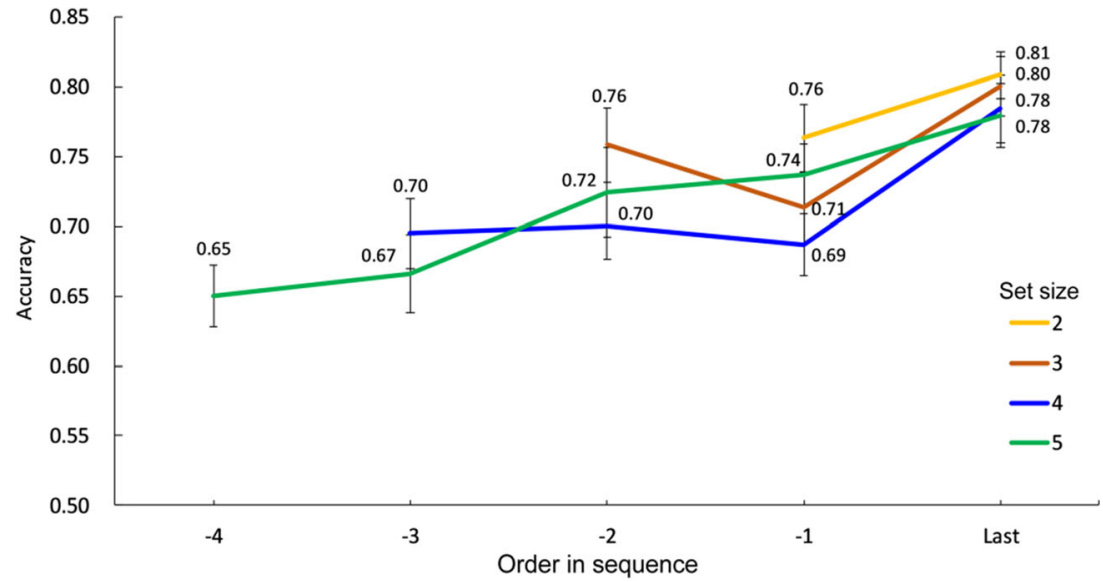



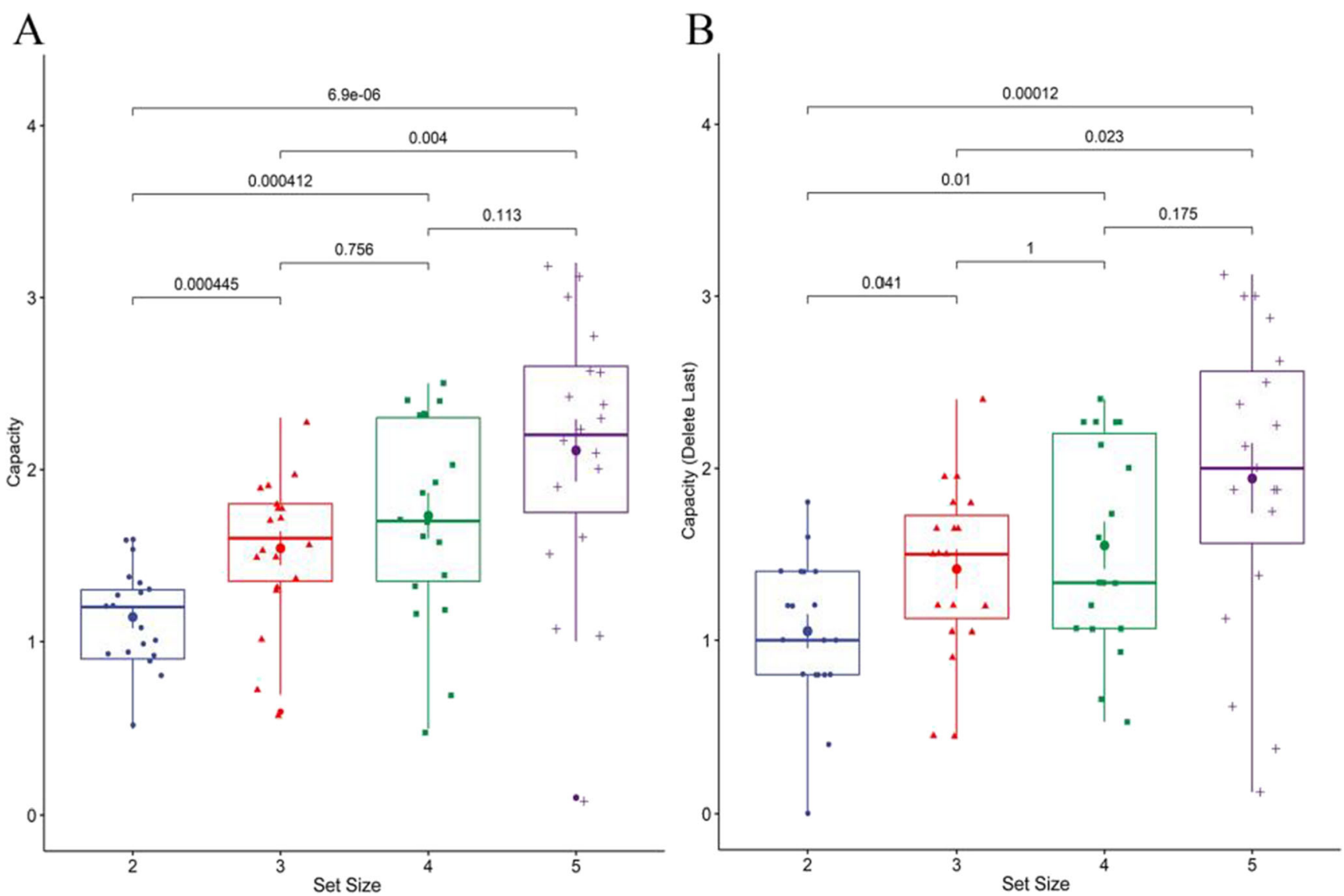

Fig. 9 (A) Capacity of four set size conditions in Experiment 2. (B) Capacity of four set size conditions with the last item excluded in Experiment 2. The error bars represent standard error of the mean

remembered significantly more accurately than the preceding frequencies. Accuracy in the recall of frequencies significantly decreased with the increase of the sequence length. All these results suggested that vtWM resources allocation to each new frequency to be remembered is affected by sequence length and serial order dynamically. However, the sequence length has no significant effect on the accuracy of the last item in the sequence. This may be caused by the quickly decaying memory storage concerning the frequency of vibrotactile stimulation. Previous studies of tactile memory suggested that sensory storage for passively presented tactile stimuli is a quickly decaying memory system, which suffers from a rapid decline in a few seconds (Bliss et al. 1966; Gallace et al. 2008). The stored frequencies may be rapidly decaying in memory, thus new frequency can be better recalled. And if storage of frequencies before the last one is decaying in a few seconds, then there may be sufficient WM resources to store the last frequency.

Third, our results showed that the vtWM capacity does not depend on whether sequentially presented vibrotactile frequencies are delivered to the same or to different fingers. Previous studies have shown that stimulus-selective activity for vibrotactile frequency is found in the PFC and somatosensory cortex (Hernández et al. 2010; Romo et al. 2012; Schmidt et al. 2017). Additionally, previous theories of WM have suggested that when different individual stimuli were retained by detailed representations in the sensory cortex and coarser representations in anterior regions, interference among stimuli representations might decrease (Christophel et al. 2017). Our data indicate that frequencies delivered sequentially to the same finger or to different fingers have equal interference in memory representations. These results indicated that WM for analog and one-dimensional frequency information is likely to be retained mainly by coarser representations in the PFC.

There are still unresolved questions with the processing and storage of vtWM for frequency. Further studies can compare different forms of stimuli presentation of vtWM. There are two basic forms of stimuli presentation in WM: simultaneous and sequential. Previous visuospatial WM studies showed that visuospatial WM system can process simultaneously presented information (Luck and Vogel 1997). Studies of tactile memory for location also showed that participants can remember positions presented simultaneously to their fingertips (Auvray et al. 2011; Bliss et al. 1966; Gallace and Spence 2009). Further studies can test the processing and storage of simultaneous presentation of vtWM. Studies can also test the underlying mechanisms of simultaneous and sequential presentation of vibrotactile frequencies using imaging tools in the further. 
In conclusion, this study shows that vtWM has a multiitem capacity, and the processing and storage of sequentially presented vibrotactile frequency information show similar characteristics to that of previous visuospatial WM studies. These results lay the foundations for comparing the vtWM to other sub-systems of WM. The results also showed that the vtWM capacity does not depend on whether sequentially presented vibrotactile frequencies are delivered to the same or to different fingers, which indicates that vtWM for frequency information is likely to be retained mainly by coarser representations in the PFC. Vibrotactile sensation belongs to the tactile sensation, which is one of the most important ways for humans to obtain external information and interact with the outside world. With the rapid development technology, tactile vibration is becoming more widely used to obtain and communicate simple information, and assist visual and auditory information processing. The present study explores the processing and storage of vibrotactile information, which improves our understanding of cognitive processing of tactile vibration and assists its applications.

Funding This work was supported by National Natural Science Foundation of China (NSFC) under Grant No. 31900751 and 31671077, and China Postdoctoral Science Foundation (2019 T120061), National Key R\&D Program of China(2019YFA0709503).

Data Availability The datasets generated or analyzed during the current study is available from the corresponding author on reasonable request.

\section{Declarations}

Consent to Participate Informed consent was obtained from all individual participants included in the study.

Consent to Publish Patients signed informed consent regarding publishing their data.

Conflict of Interest The authors declared no potential conflicts of interest.

Open Access This article is licensed under a Creative Commons Attribution 4.0 International License, which permits use, sharing, adaptation, distribution and reproduction in any medium or format, as long as you give appropriate credit to the original author(s) and the source, provide a link to the Creative Commons licence, and indicate if changes were made. The images or other third party material in this article are included in the article's Creative Commons licence, unless indicated otherwise in a credit line to the material. If material is not included in the article's Creative Commons licence and your intended use is not permitted by statutory regulation or exceeds the permitted use, you will need to obtain permission directly from the copyright holder. To view a copy of this licence, visit http://creativecommons.org/licenses/by/4.0/.

\section{References}

Auvray, M., Gallace, A., \& Spence, C. (2011). Tactile short-term memory for stimuli presented on the fingertips and across the rest of the body surface. Attention Perception \& Psychophysics, 73(4), 1227-1241. https://doi.org/10.3758/s13414-011-0098-6

Bancroft, T. D., Hockley, W. E., \& Servos, P. (2012). Can vibrotactile working memory store multiple items? Neuroscience Letters, 514(1), 31-34. https://doi.org/10.1016/j.neulet.2012.02.044

Bliss, J. C., Crane, H. D., Mansfield, P. K., \& Townsend, J. T. (1966). Information available in brief tactile presentations. Perception \& Psychophysics, 1, 273-283. https://doi.org/10.3758/BF03207391

Christophel, T. B., Klink, P. C., Spitzer, B., Roelfsema, P. R., \& Haynes, J. D. (2017). The distributed nature of working memory. Trends in Cognitive Sciences, 21(2), 111-124. https://doi.org/10.1016/j.tics.2016.12.007

Cowan, N. (2001). The magical number 4 in short-term memory: A reconsideration of mental storage capacity. Behavioral and Brain Sciences, 24, 87-114. https://doi.org/10.1017/s0140525x01003922

Cowan, N. (2010). The magical mystery four how is working memory capacity limited, and why? Current Directions in Psychological Science, 19(1), 51-57. https://doi.org/10.1177/0963721409359277

Gallace, A., Tan, H. Z., Haggard, P., \& Spence, C. (2008). Short term memory for tactile stimuli. Brain Research, 1190, 132-142. https:// doi.org/10.1016/j.brainres.2007.11.014

Gallace, A., \& Spence, C. (2009). The cognitive limitations and neural correlates of tactile memory. Psychological Bulletin, 135, 380-406. https://doi.org/10.1037/a0015325

Gorgoraptis, N., Catalao, R. F. G., Bays, P. M., \& Husain, M. (2011). Dynamic updating of working memory resources for visual objects. Journal of Neuroscience, 31(23), 8502-8511. https://doi.org/10. 1523/JNEUROSCI.0208-11.2011

Harris, J. A., Harris, I. M., \& Diamond, M. E. (2001). The topography of tactile working memory. Journal of Neuroscience, 21(20), 82628269. https://doi.org/10.1523/JNEUROSCI.21-20-08262.2001

Harris, J. A., Miniussi, C., Harris, I. M., \& Diamond, M. E. (2002). Transient storage of a tactile memory trace in primary somatosensory cortex. Journal of Neuroscience, 22(19), 8720-8725. https:// doi.org/10.1523/JNEUROSCI.22-19-08720.2002

Hernández, A., Nácher, V., Luna, R., Zainos, A., Lemus, L., Alvarez, M., Vázquez, Y., Camarillo, L., \& Romo, R. (2010). Decoding a perceptual decision process across cortex. Neuron, 66(2), 300-314. https://doi.org/10.1016/j.neuron.2010.03.031

Iwamura, Y., Tanaka, M., Sakamoto, M., \& Hikosaka, O. (1993). Rostrocaudal gradients in the neuronal receptive field complexity in the finger region of the alert monkey's postcentral gyrus. Experimental Brain Research, 92(3), 360-368. https://doi.org/10. 1007/BF00229023

Luck, S. J., \& Vogel, E. K. (1997). The capacity of visual working memory for features and conjunctions. Nature, 390(6657), 279-281.

Merzenich, M. M., Kaas, J. H., Sur, M., \& Lin, C. (1978). Double representation of the body surface within cytoarchitectonic area $3 \mathrm{~b}$ and 1 in "si" in the owl monkey (aotus trivirgatus). Journal of Comparative Neurology, 181(1), 41-73. https://doi.org/10.1002/ cne.901810104

Romo, R., Brody, C. D., Hernández, A., \& Lemus, L. (1999). Neuronal correlates of parametric working memory in the prefrontal cortex. Nature, 399(6735), 470-473. https://doi.org/10.1038/20939

Romo, R., \& Salinas, E. (2003). Flutter discrimination: Neural codes, perception, memory and decision making. Nature Reviews Neuroscience, 4, 203-218. https://doi.org/10.1038/nrn1058

Romo, R., Lemus, L., \& Lafuente, V. D. (2012). Sense, memory, and 
decision-making in the somatosensory cortical network. Current Opinion in Neurobiology, 22(6), 914-919. https://doi.org/10.1016/ j.conb.2012.08.002

Schmidt, T. T., Wu, Y. H., \& Blankenburg, F. (2017). Content-specific codes of parametric vibrotactile working memory in humans. Journal of Neuroscience the Official Journal of the Society for Neuroscience, 37(40), 9771-9777. https://doi.org/10.1523/ JNEUROSCI.1167-17.2017

Spitzer, B., \& Blankenburg, F. (2011). Stimulus-dependent EEG activity reflects internal updating of tactile working memory in humans. Proceedings of the National Academy of Sciences, 108(20), 8444 8449. https://doi.org/10.1073/pnas.1104189108
Wang, C., \& Wang, D. (2021). Capacity and maintenance mechanism of vibrotactile working memory. Advances in Psychological Science, 29(7), 1141-1148. https://doi.org/10.3724/SP.J.1042.2021.01141

Zhao, D., Zhou, Y. D., Bodner, M., \& Ku, Y. (2017). The causal role of the prefrontal cortex and somatosensory cortex in tactile working memory. Cerebral Cortex, 28(10), 3468-3477. https://doi.org/10. 1093/cercor/bhx213

Publisher's Note Springer Nature remains neutral with regard to jurisdictional claims in published maps and institutional affiliations. 